\title{
Novel spectrophotometric method for determination of cinacalcet hydrochloride in its tablets via derivatization with 1,2-naphthoquinone-4-sulphonate
}

\author{
Ibrahim A Darwish*, Mona M Al-Shehri and Manal A El-Gendy
}

\begin{abstract}
This study represents the first report on the development of a novel spectrophotometric method for determination of cinacalcet hydrochloride (CIN) in its tablet dosage forms. Studies were carried out to investigate the reaction between CIN and 1,2-naphthoquinone-4-sulphonate (NQS) reagent. In alkaline medium (pH 8.5), an orange redcolored product exhibiting maximum absorption peak $\left(\lambda_{\max }\right)$ at $490 \mathrm{~nm}$ was produced. The stoichiometry and kinetic of the reaction were investigated and the reaction mechanism was postulated. This color-developing reaction was employed in the development of a simple and rapid visible-spectrophotometric method for determination of CIN in its tablets. Under the optimized reaction conditions, Beer's law correlating the absorbance with CIN concentration was obeyed in the range of $3-100 \mu \mathrm{g} / \mathrm{ml}$ with good correlation coefficient (0.9993). The molar absorptivity $(\varepsilon)$ was $4.2 \times 10^{5} \mathrm{l} / \mathrm{mol} / \mathrm{cm}$. The limits of detection and quantification were $1.9 \mathrm{and} 5.7 \mathrm{\mu g} / \mathrm{ml}$, respectively. The precision of the method was satisfactory; the values of relative standard deviations (RSD) did not exceed $2 \%$. No interference was observed from the excipients that are present in the tablets. The proposed method was applied successfully for the determination of CIN in its pharmaceutical tablets with good accuracy and precisions; the label claim percentage was $100.80-102.23 \pm 1.27-1.62 \%$. The results were compared favorably with those of a reference pre-validated method. The method is practical and valuable in terms of its routine application in quality control laboratories.
\end{abstract}

Keywords: Cinacalcet HCl, 1,2-Naphthoquinone-4-sulphonate, Kinetic, Spectrophotometry, Pharmaceutical analysis

\section{Background}

Cinacalcet hydrochloride (CIN); N-[1-(R)-(-)-(1-naphthyl) ethyl]-3-[3-(trifluoromethyl) phenyl]-1-aminopropane $\mathrm{HCl}$ is a selective calcimimetic agent, which acts on a calciumsensing receptor of the parathyroid gland. This principal negative regulator of parathyroid hormone release increases its selectivity to activation by extracellular calcium, thus decreasing the parathyroid hormone levels $[1,2]$. CIN is effective in clinical setting and it has been approved for the treatment of secondary hyperthyroidism in patients with chronic kidney disease placed on dialysis [3], and for the treatment of elevated calcium levels in patients with parathyroid carcinoma [4].

\footnotetext{
* Correspondence: idarwish@ksu.edu.sa

Department of Pharmaceutical Chemistry, College of Pharmacy, King Saud University, P.O. Box 2457, Riyadh 11451, Saudi Arabia
}

The effective and safe therapy with CIN is basically depending on the quality of its pharmaceutical preparations (tablets), and assessing its concentrations in tablets for the purposes of quality control. Literature review revealed that there were only two methods were employed for thin-layer [5] and liquid chromatographic $[5,6]$ enantiomeric separation of CIN enantiomers in laboratory-made racemic mixtures. However, no reports have been found for describing the development of analytical methods for the quantitative determination of CIN in its tablets. Therefore, this study was devoted to the development of a new method for determination of CIN in its tablets for the purpose of its pharmaceutical quality control.

Spectrophotometry is considered the most convenient technique because of its inherent simplicity, low cost 
and wide availability [7]. As well, 1,2-Naphthoquinone4-sulphonic sulphonate (NQS) has been used as a colordeveloping reagent in the spectrophotometric determination of many pharmaceutical amines [8-11]. The reaction between NQS and CIN has not been investigated yet. Therefore, the present study was devoted to investigate this and its employment in the development of a new simple and rapid spectrophotometric method for determination of CIN in its tablets.

\section{Experimental \\ Apparatus}

Double beam V-530 (JASCO Ltd., Kyoto, Japan) ultraviolet-visible spectrophotometer with matched $1-\mathrm{cm}$ quartz cells was used for all the spectrophotometric measurements. pH meter, model 350 (Bibby Scientific Ltd., T/As Jenway, Essex, England). MLW type thermostatically controlled water bath (Memmert GmbH Co., Schwabach, Germany).

\section{Reagents and Materials}

Cinacalcet hydrochloride (Amgen Inc., Thousand Oaks, CA, USA) was obtained and used as received; its purity was $>99 \%$. A solution of $0.5 \%(\mathrm{w} / \mathrm{v})$ of NQS (Aldrich Chemical Co., St. Louis, USA) was freshly prepared and protected from light during use. Clark and Lubs buffer solution was prepared by mixing $50 \mathrm{ml}$ of $0.2 \mathrm{M}$ aqueous solution of boric acid and potassium chloride (1 liter containing $12.368 \mathrm{~g}$ of boric acid and $14.90 \mathrm{~g}$ of potassium chloride) with $21.3 \mathrm{ml}$ of $0.2 \mathrm{M}$ sodium hydroxide in $200 \mathrm{ml}$ standard flask [12], and adjusted by $\mathrm{pH}$ meter. Tris buffer was prepared by mixing $100 \mathrm{ml}$ $0.1 \mathrm{M}$ tris(hydroxymethyl)aminomethane with $29.4 \mathrm{ml}$ of $0.1 \mathrm{M} \mathrm{HCl}$ [13]. Britton-Robinson buffer composed of $0.04 \mathrm{M}$ boric acid, $0.04 \mathrm{M}$ phosphoric acid and $0.04 \mathrm{M}$ acetic acid adjusted at $\mathrm{pH} 8.5$ by using $0.2 \mathrm{M}$ sodium hydroxide [8]. Phosphate buffer composed of $0.1 \mathrm{M}$ disodium hydrogen phosphate $(14.2 \mathrm{~g} / \mathrm{l})$ and $0.1 \mathrm{M}$ sodium hydroxide [13]. Sensipar ${ }^{\circledR}$ and Mimpara ${ }^{\circledR}$ tablets (Amgen Inc, Thousand Oaks, CA, USA) were labeled to contain $60 \mathrm{mg}$ CIN per tablet. Double distilled water was obtained through WSC-85 water purification system (Hamilton Laboratory Glass Ltd., Kent, USA) and used throughout the work. All solvents and materials used throughout this study were of analytical grade.

\section{Preparation of Solutions Standard CIN Solution}

An accurately weighed amount (50 mg) of CIN was quantitatively transferred into a 25 -ml calibrated flask, dissolved in $20 \mathrm{ml}$ distilled water, completed to volume with the same solvent to obtain a stock solution of 2 $\mathrm{mg} / \mathrm{ml}$. The stock solution was found to be stable for at least two weeks when kept in a refrigerator. The stock solution was further diluted with water to obtain working solutions in the range of $3-100 \mu \mathrm{g} / \mathrm{ml}$.

\section{Tablet Sample Solution}

Twenty tablets were weighed and finely powdered. An accurately weighed quantity of the powder equivalent to $100 \mathrm{mg}$ of the active ingredient was transferred into a $100-\mathrm{ml}$ calibrated flask, and dissolved in about $40 \mathrm{ml}$ of distilled water. The contents of the flask were swirled, sonicated for 5 minutes, and then completed to volume with water. The contents were mixed well and filtered; the first portion of the filtrate was rejected. The filtered solution was diluted quantitatively with distilled water to obtain suitable concentrations for the analysis by the proposed spectrophotometric method.

\section{General Recommended Procedures}

One milliliter of CIN solution containing $3-100 \mu \mathrm{g} / \mathrm{ml}$ was transferred into separate $10-\mathrm{ml}$ calibrated flask. A $0.5 \mathrm{ml}$ of tris buffer solution of $\mathrm{pH} 8.5$ and $1 \mathrm{ml}$ of NQS solution $(0.5 \%, \mathrm{w} / \mathrm{v})$ were added. The reaction solution was allowed to proceed for $10 \mathrm{~min}$ at room temperature $\left(25 \pm 2^{\circ} \mathrm{C}\right)$ and completed to volume with water. The resulting solution was measured at $490 \mathrm{~nm}$ against reagent blank treated similarly.

\section{Determination of Stoichiometric Ratio Job's Method}

The Job's method of continuous variation [14] was employed. Master equimolar $\left(1 \times 10^{-4} \mathrm{M}\right)$ aqueous solutions of CIN and NQS were prepared. Series of 10-ml portions of the master solutions of CIN and NQS were made up comprising different complementary proportions $(0: 10,1: 9, \ldots, 9: 1,10: 0$, inclusive) in 10-ml calibrated flasks containing $0.5 \mathrm{ml}$ of tris buffer solution ( $\mathrm{pH} 8.5$ ). The solutions were further manipulated as described under the general recommended procedures.

\section{Limiting Logarithmic Method}

The limiting logarithmic method [15] was employed. Two sets of experiments were carried out employing the general recommended procedures described above. The first set of experiments was carried using varying concentrations of the analytical reagent $\left(1.9 \times 10^{-3}-9.6 \times\right.$ $\left.10^{-3} \mathrm{M}\right)$ at a fixed CIN concentration $\left(1 \times 10^{-4} \mathrm{M}\right)$. The second set of experiments was carried using varying concentrations of CIN $\left(7.6 \times 10^{-6}-2.5 \times 10^{-4} \mathrm{M}\right)$ at a fixed concentration of NQS $\left(1.9 \times 10^{-2} \mathrm{M}\right)$. The logarithms of the obtained absorbances for the reaction of CIN with NQS were plotted as a function of the logarithms of the concentrations of the reagent and CIN in the first and second sets of experiments. The slopes of the fitting lines in both sets of experiments were calculated. 


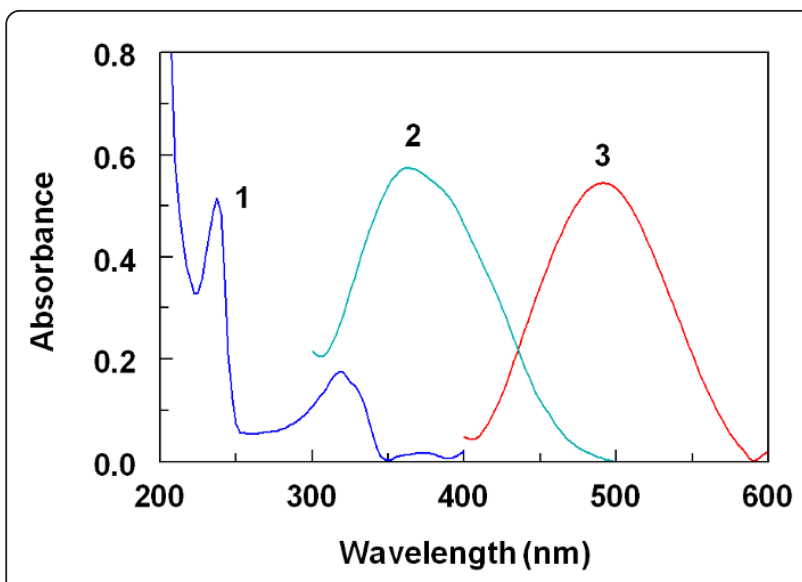

Figure 1 Absorption spectra of CIN against water (1), NQS against water (2), and their reaction product against reagent blank (3)

\section{Results and Discussion Absorption Spectrum}

The absorption spectrum of CIN was recorded against water (Figure 1). It was found that CIN exhibits two maximum absorption peaks $\left(\lambda_{\max }\right)$ at 235 and $320 \mathrm{~nm}$, and the molar absorptivities $(\varepsilon)$ at these wavelengths were $5.1 \times 10^{4}$ and $1.7 \times 10^{4} \mathrm{l} / \mathrm{ml} / \mathrm{cm}$, respectively. Because of the blue shifted $\lambda_{\max }$ of CIN, its determination in the pharmaceutical formulations based on the direct measurement of its absorption for ultraviolet light is susceptible to potential interferences from the coextracted excipients. Therefore, derivatization of CIN to a more red-shifted derivative was necessary. CIN contains secondary amino group for which many chromogenic reagents are available for color-producing reactions. These reactions include formation of colored charge-transfer complex with electron acceptor [16], formation of ion-pair associates with pairing reagents [17-19], and formation of condensation product with a chromogenic reagent [20]. These methods are usually associated with some major drawbacks such as laborious multiple extraction steps in the analysis by ion-pair based methods [17-19], or in preparation of the free base of the drug prior to the analysis by charge-transferbased methods [16], and long reaction time, thus the procedure is time-consuming [20]. Darwish et al. [21-24] has demonstrated that NQS is a valuable colordeveloping reagent in the development of simple spectrophotometric methods for the determination of many pharmaceutical amines in the form of their acid salts. For these reasons, the present study was devoted to investigate the reaction between NQS and CIN, and employed this color reaction in the development of a new simple and rapid spectrophotometric method for determination of CIN in its tablets. The reaction between CIN and NQS was performed, and the absorption spectrum of the reaction product was recorded against reagent blank. The product was orange redcolored exhibiting $\lambda_{\max }$ at $490 \mathrm{~nm}$ (Figure 1). Obviously, the $\lambda_{\max }$ of CIN-NQS derivative was red-shifted from the underivatized CIN by $171 \mathrm{~nm}$. As well, the value of $\varepsilon$ (sensitivity) was greatly enhanced to be $4.2 \times 10^{5} \mathrm{l} /$ $\mathrm{mol} / \mathrm{cm}$. The following paragraphs describe the optimization of the reaction conditions.

\section{Optimization of Reaction Conditions}

\section{Effect of NQS Concentration}

Studying the effect of NQS concentration on its reaction with CIN revealed that the reaction was dependent on the NQS concentration as the readings increased with the increase in the reagent concentration (Figure 2). The highest readings were attained at a concentration range of $0.4-0.6 \%(\mathrm{w} / \mathrm{v})$ beyond which the readings slightly decreased. A concentration of $0.5 \%(\mathrm{w} / \mathrm{v})$ was used in all the subsequent experiments.

\section{Effect of $\mathrm{pH}$ and Buffer Components}

The influence of $\mathrm{pH}$ on the reaction of CIN with NQS was investigated by carrying out the reaction in buffer solution of varying $\mathrm{pH}$ values. The results revealed that CIN has difficulty to react with NQS in acidic media (Figure 2). This was possibly due to the existence of the amino group of CIN in the form of hydrochloride salt, thus it loses its nucleophilic substitution affinity. As the $\mathrm{pH}$ increased, the readings increased rapidly, as the amino group of CIN (in the hydrochloride salt) turns into the free amino group, thus facilitating the nucleophilic substitution. The maximum readings were attained at $\mathrm{pH}$ values of 8.5. At higher $\mathrm{pH}$, sharp decrease in the readings occurred. This was attributed

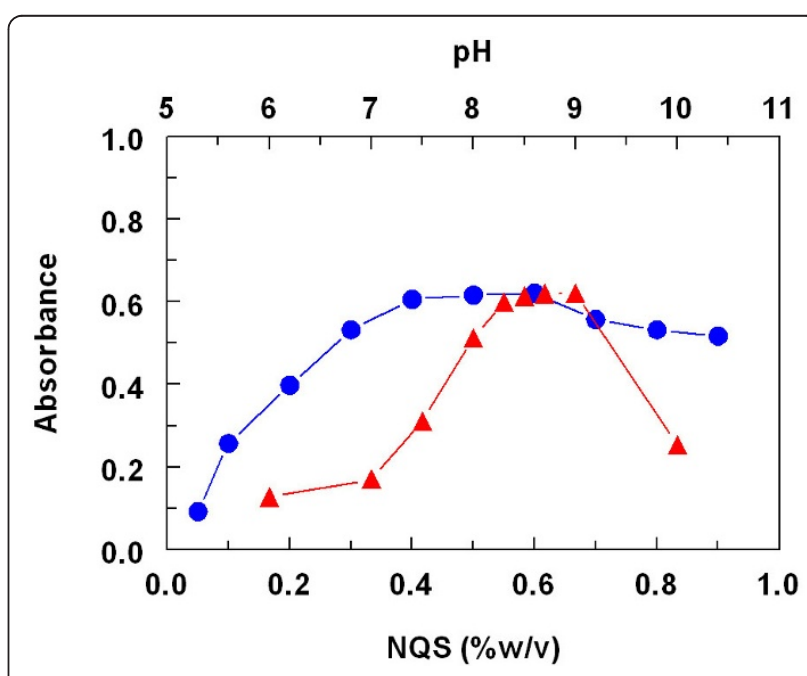

Figure 2 Effect of NQS concentration $(\bullet)$ and $\mathrm{pH}(\Delta)$ on the reaction of CIN $(70 \mu \mathrm{g} / \mathrm{ml})$ with NQS $(0.5 \%, \mathrm{w} / \mathrm{v})$. 
probably to the increase in the amount of hydroxide ion that holds back the reaction of CIN with NQS, and the instability of NQS reagent [25].

In order to investigate the effect of buffer components on the reaction, different buffer solutions of $\mathrm{pH} 8.5$ were tested: Clark, Robinson, phosphate, borate and tris buffers. The highest absorbances were obtained when tris buffer was used, thus it was used in all the subsequent experiments.

\section{Effect of Temperature and Time}

The effect of temperature on the reaction was studied by carrying out the reaction at room temperature $(25 \pm$ $\left.2^{\circ} \mathrm{C}\right)$ and at varying elevated temperatures $\left(30-60^{\circ} \mathrm{C}\right)$. The results (Figure 3 ) revealed that there was no significant difference between the readings that have been obtained at room temperature and those at elevated temperatures up to $50^{\circ} \mathrm{C}$, beyond which the readings significantly decreased. In order to establish simple analytical procedures with no need for extra equipment (water bath), further experiments were carried out at room temperature.

In order to determine the optimum time that is required for completion the reaction, it was allowed to proceed at room temperature for varying periods of time. It was found that the reaction goes to almost completion within 5 min (Figure 3), however for higher precision readings, the reaction was allowed to proceed for quite longer time; reactions in all the subsequent experiments were carried out for $10 \mathrm{~min}$.

\section{Effect of Diluting Solvent}

Upon diluting the reaction solutions with water, transparent solution was obtained indicating the solubility of the CIN-NQS product in water, and the possibility of using water as a diluting solvent. In order to select the

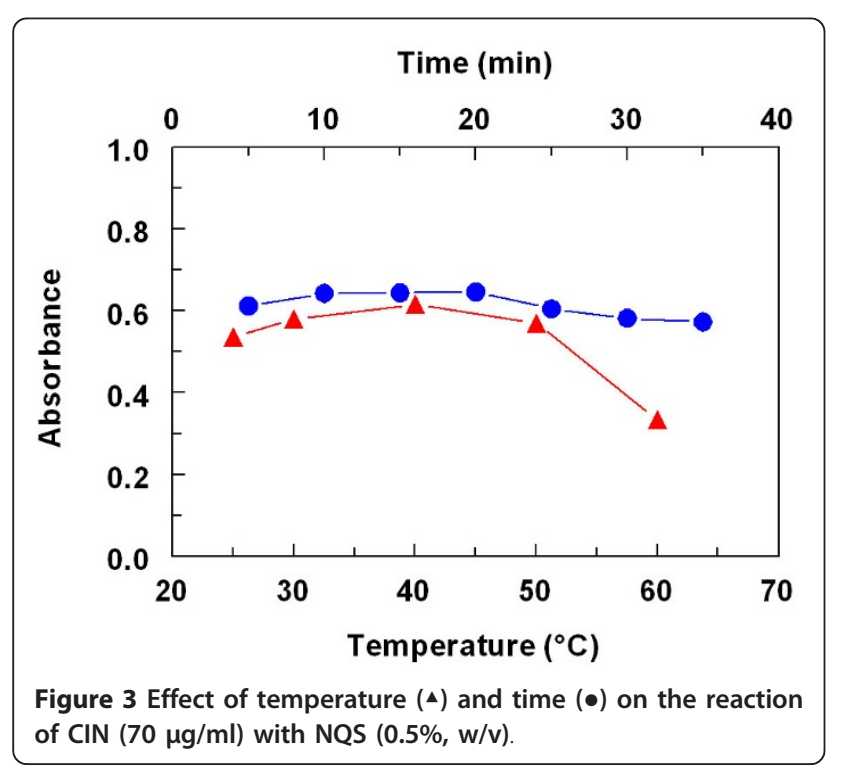

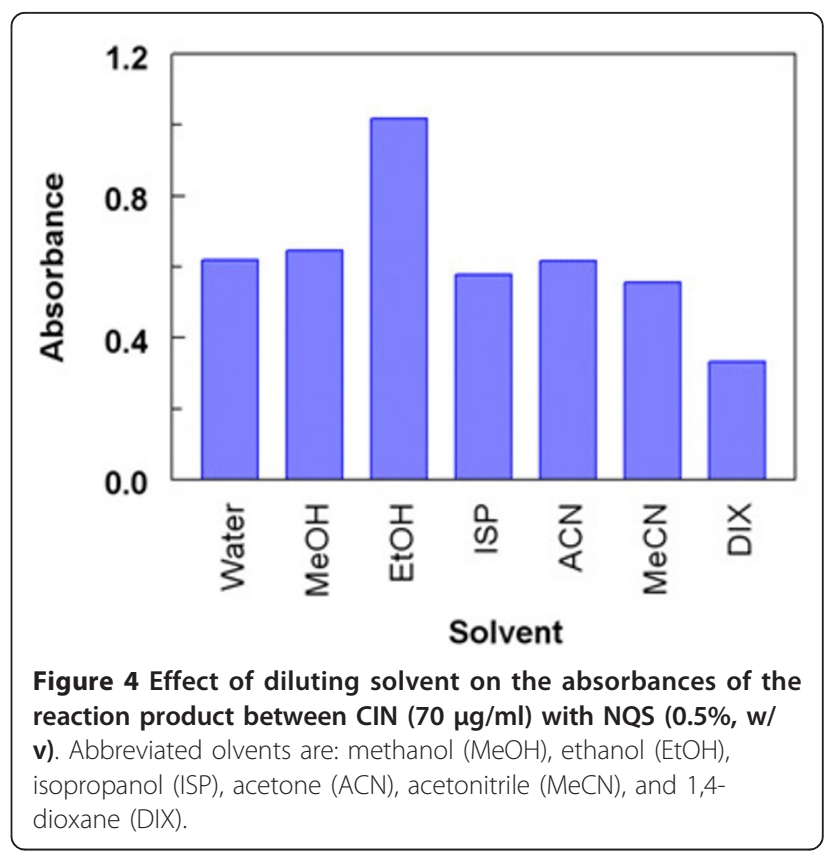

most appropriate solvent for diluting the reaction solutions, different solvents were tested and compared with water; these solvents were methanol, ethanol, isopropanol, acetone, acetonitrile, and 1,4-dioxane. The highest readings were obtained when ethanol was used for dilution (Figure 4). However, the use of organic solvents leads to high analysis cost, and more importantly, the incidence of exposure of the analysts to the side effects of these toxic solvents [26-30]. Therefore, water was used as a diluting solvent in the subsequent investigation on the expense of the higher sensitivity, which was not a main concern in the present study as the method was devoted to the analysis of CIN in its dosage forms that does not require highly sensitive assay.

\section{Stability of the Chromogen}

The effect of time on the stability of the CIN-NQS chromogen was studied by following the absorption intensity of the reaction solution (after dilution) at different time intervals. It was found that the absorbance of the chromogen remains stable for at least $1 \mathrm{~h}$. This allowed the processing of large batches of samples, and their comfortable measurements with convenience. This gives the high throughput property to the proposed method when applied for analysis of large number of samples in quality control laboratories.

\section{Stoichiometry, Kinetics and Mechanism of the Reactions}

Under the optimum conditions (Table 1), the stoichiometry of the reaction between CIN and NQS was investigated by Job's [14] and limiting logarithmic [15] methods. The symmetrical bell shape of Job's plot (not shown data) indicated that the CIN:NQS ratio was 1:1. 
Table 1 Summary for the optimization of variables affecting the reaction of CIN with NQS reagent employed in the development of the proposed spectrophotometric method

\begin{tabular}{lll}
\hline Variable & Studied range & Optimum \\
\hline NQS concentration $(\%, w / v)$ & $0.1-0.9$ & 0.5 \\
$\mathrm{pH}$ & $6-10$ & 8.5 \\
Temperature $\left({ }^{\circ} \mathrm{C}\right)$ & $25-60$ & 25 \\
Time $($ min) & $5-35$ & 10 \\
Diluting solvent & Different $^{\mathrm{a}}$ & Water \\
Measuring wavelength $(\mathrm{nm})$ & $400-600$ & 490
\end{tabular}

a solvents were: methanol, ethanol, isopropanol, acetone, acetonitrile, and 1,4dioxane.

In the limiting logarithmic method, two straight lines were obtained (Figure 5). The slopes of these lines were 1.0109 and 1.0565, confirming the 1:1 ratio for the reaction. Based on this ratio, the reaction pathway was postulated to be proceeded as shown in Figure 6.

Under the optimum conditions, the signal-time curves for the reactions at varying concentrations of CIN (1.27 $\left.\times 10^{-4}-2 \times 10^{-4} \mathrm{M}\right)$ for the reaction with a fixed concentration of NQS $\left(1.92 \times 10^{-3} \mathrm{M}\right)$ were generated. The initial reaction rates $(K)$ were determined from the slopes of these curves. The logarithms of the reaction rates $(\log K)$ were plotted as a function of logarithms of CIN concentrations $(\log C)$. A straight line with slope value of 1.0519 was obtained (Figure 7), by fitting the data to the following equation:

$$
\log K=\log K^{\prime}+\mathrm{n} \log C
$$

where $K$ is reaction rate, $K^{\prime}$ is the rate constant, $C$ is the molar concentration of CIN and $n$ (slope of

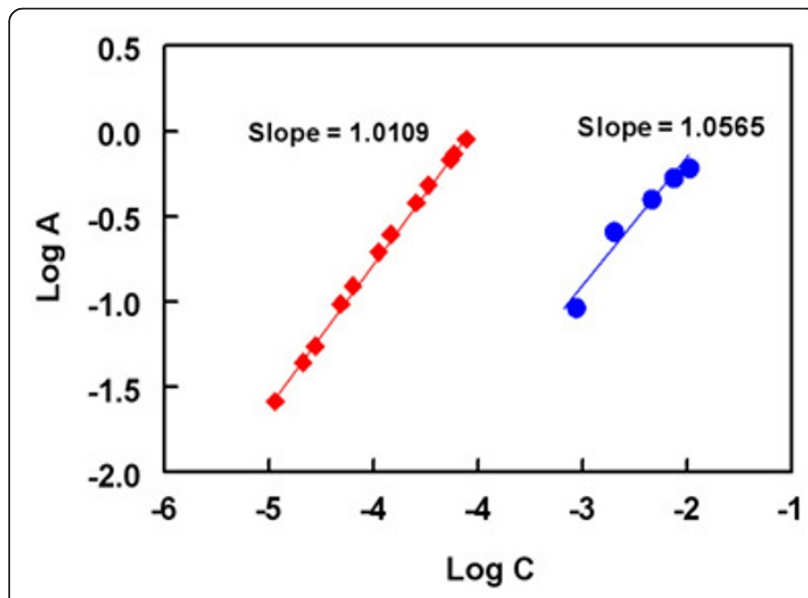

Figure 5 Limiting logarithmic plot for molar reactivity of CIN with NQS. C and A are the concentration and absorbance, respectively. For generating the first line $(\bullet)$, [NQS]: $1.9 \times 10^{-3}$; [CIN]: $7.6 \times 10^{-6}-2.5 \times 10^{-4} \mathrm{M}$. For generating the second line $(\bullet)$, [NQS]: $1.9 \times 10^{-3}-9.6 \times 10^{-3} \mathrm{M} ;[\mathrm{CIN}]: 1 \times 10^{-4} \mathrm{M}$.

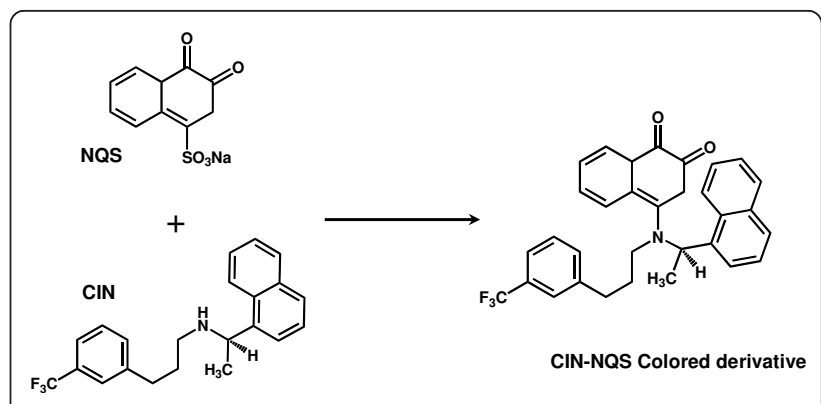

Figure 6 Scheme for the reaction pathway of CIN with NQS

regression line) is the order of the reaction. The values of the slope $(\approx 1)$ confirmed that the reaction was first order. However under the optimized reaction conditions, the concentrations of $\mathrm{NQS}$ were in much more excess than that of CIN in the reaction solution. Therefore, the reaction was regarded as pseudo-first order reaction.

\section{Validation of the Proposed Method Calibration and Sensitivity}

Under the optimum reaction conditions (Table 1), the calibration curve for the determination of CIN by its reaction with NQS was constructed by plotting the absorbances as a function of the corresponding concentrations. The regression equation for the results was $\mathrm{A}$ $=0.0092+0.0090 \mathrm{C}(\mathrm{r}=0.9993)$, where $\mathrm{A}$ is the absorbance at $490 \mathrm{~nm}, \mathrm{C}$ is the concentration of CIN in $\mu \mathrm{g} /$ $\mathrm{ml}$ in the range of $3-100 \mu \mathrm{g} / \mathrm{ml}$, and $\mathrm{r}$ is the correlation coefficient. The molar absorptivity $(\varepsilon)$ was $4.2 \times 10^{5}$ $1 / \mathrm{mol} / \mathrm{cm}$. The limit of detection (LOD) and limit of quantification (LOQ) were determined according to The International Conference of Harmonization (ICH)

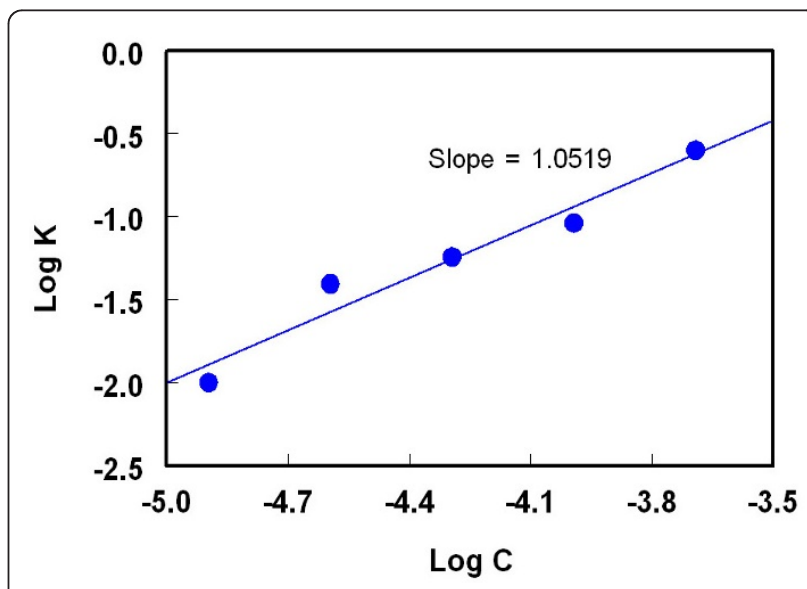

Figure 7 Linear plot for $\log C$ versus $\log K$ for the kinetic reaction of CIN with NQS. $C$ is the CIN concentration $\left(7.6 \times 10^{-6}\right.$ $\left.2.5 \times 10^{-4} \mathrm{M}\right)$ and $K$ is the reaction rate $\left(\right.$ second $\left.{ }^{-1}\right)$. 
Table 2 Parameters for the performance of the proposed spectrophotometric method for determination of CIN

\begin{tabular}{ll}
\hline Parameter & Value \\
\hline Measurement wavelength $(\mathrm{nm})$ & 490 \\
Linear range $(\mu \mathrm{g} / \mathrm{ml})$ & $3-100$ \\
Intercept & 0.0092 \\
Standard deviation of the intercept & 0.0051 \\
Slope & 0.0090 \\
Standard deviation of the slope & 0.0001 \\
Correlation coefficient $(\mathrm{r})$ & 0.9993 \\
Limit of detection, $\mathrm{LOD}(\mu \mathrm{g} / \mathrm{ml})$ & 1.9 \\
Limit of quantification, $\mathrm{LOQ}(\mu \mathrm{g} / \mathrm{ml})$ & 5.7 \\
Molar absorptivity, $\varepsilon(\mathrm{l} / \mathrm{mol} / \mathrm{cm})$ & $4.2 \times 10^{5}$ \\
\hline
\end{tabular}

guidelines for validation of analytical procedures [31]. The following formula was used: $\mathrm{LOD}$ or $\mathrm{LOQ}=\times \mathrm{SDa} /$ $\mathrm{b}$, where $x=3.3$ for LOD and 10 for LOQ, SDa is the standard deviation of the intercept, and $\mathrm{b}$ is the slope. The LOD and LOQ were found to be 1.9 and $5.7 \mu \mathrm{g} / \mathrm{ml}$, respectively. The parameters for the analytical performance of the proposed method are summarized in Table 2.

\section{Precision}

The intra-assay precision of the proposed method was determined on samples of drug solutions at varying concentration levels (Table 3 ) by analyzing 5 replicates of each sample as a batch in a single assay run. The interassay precision was determined by analyzing the same samples as duplicates in three consecutive days. The relative standard deviations (RSD) did not exceed 0.78\% (Table 3) proving the high precision of the proposed method for the routine application in the analysis of $\mathrm{CIN}$ in quality control laboratories.

\section{Accuracy and Interference Liabilities}

The accuracy of the proposed method was evaluated by the recovery studies for added concentrations. The recovery values were $98.60-99.96 \pm 0.01-0.21 \%$ (Table 4 ), indicating the accuracy of the proposed method. Before proceeding with the analysis of CIN in its dosage forms, interference liabilities were carried out to explore the effect of inactive ingredients that might be added during formulation [32]. Samples were prepared by mixing known amount $(30 \mathrm{mg}$ ) of CIN with $50 \mathrm{mg}$ of pregelatinized starch, $50 \mathrm{mg}$ of microcrystalline cellulose,

Table 3 Precision of the proposed assay at different CIN concentrations

\begin{tabular}{lll}
\hline Concentration $(\boldsymbol{\mu g} / \mathbf{m l})$ & \multicolumn{2}{l}{ Relative standard deviation } \\
\cline { 2 - 3 } & Intra-assay, $\mathbf{n}=\mathbf{5}$ & Inter-assays, $\mathbf{n = 6}$ \\
\hline 10 & 0.83 & 0.79 \\
40 & 0.83 & 0.71 \\
80 & 0.35 & 0.44 \\
\hline
\end{tabular}

Table 4 Recovery studies for determination of CIN by the proposed method

\begin{tabular}{llll}
\hline $\begin{array}{l}\text { Sample } \\
\text { number }\end{array}$ & \multicolumn{2}{c}{ CIN } & $\begin{array}{l}\text { Recovery } \\
(\% \pm \mathbf{S D})^{\mathbf{a}}\end{array}$ \\
\cline { 2 - 3 } & $\begin{array}{l}\text { Added } \\
(\boldsymbol{\mu g} / \mathrm{ml})\end{array}$ & $\begin{array}{l}\text { Found } \\
\left(\boldsymbol{\mu} \mathbf{g} / \mathbf{m l}^{\mathbf{a}}\right)^{2}\end{array}$ \\
\hline 1 & 10 & 9.86 & $98.60 \pm 0.12$ \\
2 & 20 & 19.85 & $99.25 \pm 0.21$ \\
3 & 40 & 39.95 & $99.88 \pm 0.12$ \\
4 & 80 & 79.93 & $99.91 \pm 0.01$ \\
5 & 100 & 99.96 & $99.96 \pm 0.04$ \\
\hline
\end{tabular}

${ }^{\text {a }}$ Values are mean of three determinations.

$10 \mathrm{mg}$ of povidone, $10 \mathrm{mg}$ crospovidone, $10 \mathrm{mg}$ of colloidal silicon dioxide and magnesium stearate. These laboratory-prepared samples were analyzed by the proposed method applying the general recommended procedure. The average recovery value was of $100.57 \pm$ $1.51 \%$ (Table 5). These data confirmed the absence of interference from any of the inactive ingredients with the determination of CIN by the proposed method.

\section{Robustness and Ruggedness}

Robustness was examined by evaluating the influence of small variation in the method variables on its analytical performance. In these experiments, one parameter was changed whereas the others were kept unchanged, and the recovery percentage was calculated each time. It was found that small variation in the method variables did not significantly affect the procedures; recovery values were $98.87-101.41 \pm 0.26-0.86 \%$ (Table 6 ). This indicated the reliability of the proposed method during its routine application for the analysis of CIN.

Ruggedness was also tested by applying the proposed methods to the assay of CIN using the same operational conditions but using two different instruments at two different laboratories and different elapsed time. Results obtained from lab-to-lab and day-to-day variations were reproducible, as the relative standard deviations (RSD) did not exceed $2 \%$.

Table 5 Analysis of CIN in presence of the excipients that are present in its tablets by the proposed method

\begin{tabular}{ll}
\hline Excipient & Recovery $(\% \pm \mathrm{SD})^{\mathrm{a}}$ \\
\hline Pregelatinized starch $(50)^{\mathrm{b}}$ & $102.09 \pm 0.26$ \\
Microcrystalline cellulose (50) & $102.0 \pm 0.57$ \\
Povidone (10) & $99.28 \pm 0.27$ \\
Cross povidone (10) & $99.25 \pm 0.25$ \\
Colloidal silicon dioxide (10) & $98.34 \pm 0.26$ \\
Magnesium stearate (10) & $101.16 \pm 0.95$ \\
\cline { 2 - 2 } Average \pm SD & $100.57 \pm 1.51$ \\
${ }^{\text {a }}$ Values are mean of three determinations. \\
${ }^{b}$ Figures in parenthesis are the amounts in mg added per $30 \mathrm{mg}$ of CIN.
\end{tabular}


Table 6 Influence of small variations in the assay conditions on the analytical performance of the proposed spectrophotometric method for determination of CIN using NQS reagent

\begin{tabular}{ll}
\hline Parameters & Recovery $(\% \pm \text { SD })^{\mathbf{a}}$ \\
\hline Recommended conditions ${ }^{\mathrm{b}}$ & $100.57 \pm 1.51$ \\
NQS concentration $(\%, \mathrm{w} / \mathrm{v})$ & $100.06 \pm 0.53$ \\
0.4 & $100.20 \pm 0.34$ \\
0.6 & \\
Buffer solution $(\mathrm{pH})$ & $98.95 \pm 0.67$ \\
8.3 & $100.55 \pm 0.68$ \\
9.7 & \\
Temperature $\left({ }^{\circ} \mathrm{C}\right)$ & $99.24 \pm 1.04$ \\
20 & $101.15 \pm 0.48$ \\
30 & \\
Reaction time $(\mathrm{min})$ & $99.87 \pm 0.26$ \\
5 & $101.41 \pm 0.66$ \\
15 &
\end{tabular}

${ }^{a}$ Values are mean of 3 determinations.

${ }^{b}$ The recommended conditions are given in the Experimental Section.

\section{Application of the Proposed Method to Analysis of CIN in} Tablets

It is evident from the above-mentioned results that the proposed method gave satisfactory results with CIN in bulk. Thus its pharmaceutical dosage forms (Sensipar ${ }^{\mathbb{R}}$ and Mimpara ${ }^{\mathbb{R}}$ tablets) were subjected to the analysis of their CIN contents by the proposed and reference method. Since there was neither official method nor reported spectrophotometric method for the quantitative determination of CIN in its tablets, a pre-validated chromatographic method developed in our laboratory was used as a reference method [Khalil NY, Wani TA, Darwish IA: An ICH-validated high-performance liquid chromatographic method with fluorescence detection for determination of cinacalcet hydrochloride in tablets and plasma, unpublished]. The label claim percentages were $102.23 \pm 1.27$ and $100.80 \pm 1.60 \%$ for Sensipar ${ }^{\circledR}$ and Mimpara ${ }^{\circledR}$ tablets, respectively (Table 7). This result was compared with that obtained from the reference

Table 7 Determination of CIN in tablets by the proposed and reference methods

\begin{tabular}{|c|c|c|c|c|}
\hline \multirow[t]{2}{*}{ Tablets $^{a}$} & \multicolumn{2}{|c|}{ Recovery $(\% \pm S D)^{b}$} & \multirow[t]{2}{*}{$t$-value ${ }^{c}$} & \multirow[t]{2}{*}{ F-value $^{c}$} \\
\hline & $\begin{array}{l}\text { Proposed } \\
\text { method }\end{array}$ & $\begin{array}{l}\text { Reference } \\
\text { method }^{d}\end{array}$ & & \\
\hline Sensipar & $102.23 \pm 1.27$ & $101.43 \pm 1.35$ & 0.97 & 1.14 \\
\hline Mimpara & $100.80 \pm 1.60$ & $100.10 \pm 1.30$ & 1.71 & 1.91 \\
\hline
\end{tabular}

a Labeled to contain $60 \mathrm{mg}$ CIN per tablet.

${ }^{b}$ Values are mean of five determinations.

c The tabulated t- and F-values at 95\% confidence limit are 2.31 and 6.61, respectively.

${ }^{d}$ Reference method: Khalil NY, Wani TA, Darwish IA: An ICH-validated highperformance liquid chromatographic method with fluorescence detection for determination of cinacalcet hydrochloride in tablets and plasma, unpublished. method by statistical analysis with respect to the accuracy (by $t$-test) and precision (by $F$-test). No significant differences were found between the calculated and theoretical values of $t$ - and $F$-tests at 95\% confidence level proving similar accuracy and precision in the determination of CIN by both methods.

\section{Conclusions}

The present study described, for the first time, the successful evaluation of NQS as an analytical reagent in the development of simple and rapid spectrophotometric method for the accurate determination of CIN in its dosage forms. The method described herein has many advantages: it does not need expensive sophisticated apparatus, it is simple and rapid, and it has high sensitivity. The proposed method used inexpensive reagents with excellent shelf life, and is available in any analytical laboratory. Therefore, the method is practical and valuable for its routine application in quality control laboratories for analysis of CIN.

\section{Abbreviations}

CIN: cinacalcet HCl; NQS: 1,2-naphthoquinone-4-sulphonate; wavelength of maximum absorption, $\lambda_{\max }$ : molar absorptivity: $\varepsilon$; ICH: The international Conference on Harmonization; LOD: limit of detection; LOQ: limit of quantification; SD: standard deviation; RSD: relative standard deviation.

\section{Acknowledgements}

The authors extend their appreciation to the Deanship of Scientific Research at King Saud University for funding the work through the research group No. RGP-VPP-065.

\section{Authors' contributions}

IAD proposed the subject, designed the study, participated in the results discussion and revised the manuscript. MMA participated in the assay design, results discussion, and preparing the manuscript. MAE conducted the optimization of the assay conditions and its validation and prepared the draft version of the manuscript.

\section{Competing interests}

The authors declare that they have no competing interests.

Received: 12 November 2011 Accepted: 3 February 2012 Published: 3 February 2012

\section{References}

1. Franceschini N, Joy MS, Kshirsagar A: Cinacalcet $\mathrm{HCl}$ : a calcimimetic agent for the management of primary and secondary hyperparathrodism. Exp Opin Invest Drugs 2003, 2:1413-1421.

2. Torres PU: Cinacalcet $\mathrm{HCl}$ : a novel treatment for secondary hyperparathyroidism caused by chronic kidney disease. J Ren Nut 2006, 16:253-258.

3. Amgen: Sensipar for Parathyroid Carcinoma. Amgen 2009 [http://www. sensipar.com/Sensipar_for_HPC.html].

4. Block GA, Martin KJ, de Francisco AL: Cinacalcet for secondary hyperparathyroidism in patients receiving hemodialysis. $N$ Engl I Med 2004, 350:1516-1525.

5. Bhushan R, Dubey R: Indirect reversed-phase high-performance liquid chromatographic and direct thin-layer chromatographic enantioresolution of (R, S)-cinacalcet. Biomed Chromatogr 2011, 25:674-679.

6. Ravinder V, Ashok S, Varma MS, Babu CVR, Shanker K, Balaswam G: A validated chiral LC method for the enantiomeric separation of cinacalcet hydrochloride. Chromatographia 2009, 70:229-232. 
7. Görög S: Ultraviolet-Visible Spectrophotometry in Pharmaceutical Analysis CRC Press, New York; 1994.

8. Kumar CHA, Kumar TA, Gurupadayya BM, Sloka SN, Reddy BMR: Novel spectrophotometric determination of valacyclovir and cefotaxime using 1, 2-napthaquinone-4-sulfonic acid sodium in bulk and pharmaceutical dosage form. Arch Appl Sci Res 2010, 2:278-287.

9. Mahmoud AM, Khalil NY, Darwish IA, Aboul-Fadl Y: Selective spectrophotometric and spectrofluorometric methods for the determination of amantadine hydrochloride in capsules and plasma via derivatization with 1,2-naphthoquinone-4-sulphonate. Int J Anal Chem 2009, 2009:8, Article ID 810104

10. Padmarajaiah N, Kumar HRA, Vasantha RA, Yathirajan HS: Novel reagents for the sensitive spectrophotometric determination of flutamide, an anticancer drug in pharmaceutical preparations. Int J Pharm 2002, 235:113-120.

11. Al-Momani IF: Spectrophotometric determination of selected cephalosporins in drug formulations using flow injection analysis. J Pharm Biomed Anal 2001, 25:751-757.

12. Pesez M, Bartos J: Colorimetric and fluorimetric analysis of organic compounds and drugs Marcel Dekker Inc., New York; 1974, 628-630.

13. Robinson RA, Stokes RH: Electrolyte solutions, the measurement and interpretation of conductance, chemical potential, and diffusion in solutions of simple electrolytes. 2 edition. London, Butterworths; 1968.

14. Job P: Advanced Physicochemical Experiments. 2 edition. Oliner and Boyd Edinburgh; 1964, 54.

15. Rose J: Advanced Physicochemical Experiments Pitman, London; 1964

16. Foster R: Organic charge-transfer complexes London, New York, Academic Press; 1969, 470.

17. Starczewska B, Jasińska A, Białous B: Study and analytical application of ion-pair formation in the system fluoxetine-pyrocatechol violet and fluvoxamine-pyrocatechol violet. Pharmazie 2003, 58:245-248.

18. Starczewska B, Mielech K: Application of chrome azurol S for the extractive spectrophotometric determination of fluoxetine and fluvoxamine. J Pharm Biomed Anal 2000, 23:243-247.

19. Starczewska B, Puzanowska-Tarasiewicz H, Baranowska K: Investigation and analytical application of the reactions of eriochrome cyanine $\mathrm{R}$ with fluvoxamine and fluoxetine. J Pharm Biomed Anal 2000, 23:477-481.

20. Onal A, Kepekçi SE, Oztunç AA: Spectrophotometric methods for the determination of the antidepressant drug paroxetine hydrochloride in tablets. J AOAC Int 2005, 88:490-495.

21. Darwish IA: Kinetic spectrophotometric methods for determination of trimetazidine dihydrochloride. Anal Chim Acta 2005, 551:222-231.

22. Darwish IA, Abdine HH, Amer SM, Al-Rayes LI: Simple spectrophotometric method for the determination of paroxetine in tablets using 1,2naphthoquinone-4-sulphonate as a chromogenic reagent. Int I Anal Chem 2009, 2009:8, Article ID 237601.

23. Darwish IA, Abdine HH, Amer SM, Al-Rayes LI: New spectrophotometric and fluorimetric methods for determination of fluoxetine in pharmaceutical formulations. Int J Anal Chem 2009, 2009:9, Article ID 257309.

24. Darwish IA, Abdine HH, Amer SM, Al-Rayes LI: Spectrophotometric Study for the Reaction of Fluvoxamine 1,2-naphthoquinone-4-sulphonate: Kinetic, Mechanism, and Use for Determination of Fluvoxamine in its Dosage Forms. Spectrochim Acta A 2009, 72:897-902

25. Saurina J, Hernandez-Cassou S: Continuous-flow spectrophotometric determination of amino acids with 1,2-naphthoquinone-4-sulphonate reagent. Anal Chim Acta 1993, 283:414-420.

26. Fidler AT, Baker EL, Letz RE: Neurobehavioural effects of occupational exposure to organic solvents among construction painters. $\mathrm{Br} J$ Indust Med 1987, 44:292-308.

27. Kristensen P, Hilt B, Svendsen K, Grimsrud TK: Incidence of lymphohaematopoietic cancer at university laboratory: a cluster investigation. Eur J Epidemiol 2008, 23:11-15.

28. Lindbohm ML, Taskinen HT, Sallman M, Hemminki K: Spontaneous abortions among women exposed to organic solvents. Am J Indust Med 2007, 17:449-463.

29. Wennborg $H$, Bonde JP, Stenbeck M, Olsen J: Adverse reproduction outcomes among employee in biomed-ical research laboratories. Scand J Work Environ Health 2002, 28:5-11.
30. Wennborg H, Lennart B, Harri V, Gösta A: Pregnancy outcome of personnel in swedish biomedical re-search laboratories. J Occup Environ Med 2000, 42:438-446.

31. International Conference on Harmonisation Q2(R1): Validation of analytical procedures: text and methodology. London 2005 [http://www.ich.org/ fileadmin/Public_Web_Site/ICH_Products/Guidelines/Quality/Q2_R1/Step4/ Q2_R1_Guideline.pdf].

32. Amgen: Sensipar ${ }^{\circledast}$ (cinacalcet) Tablets. Amgen Inc., One Amgen Center Drive, Thousand Oaks, CA; 2010:V6:91320-1799.

doi:10.1186/1752-153X-6-11

Cite this article as: Darwish et al:: Novel spectrophotometric method for determination of cinacalcet hydrochloride in its tablets via derivatization with 1,2-naphthoquinone-4-sulphonate. Chemistry Central Journal 2012 6:11.

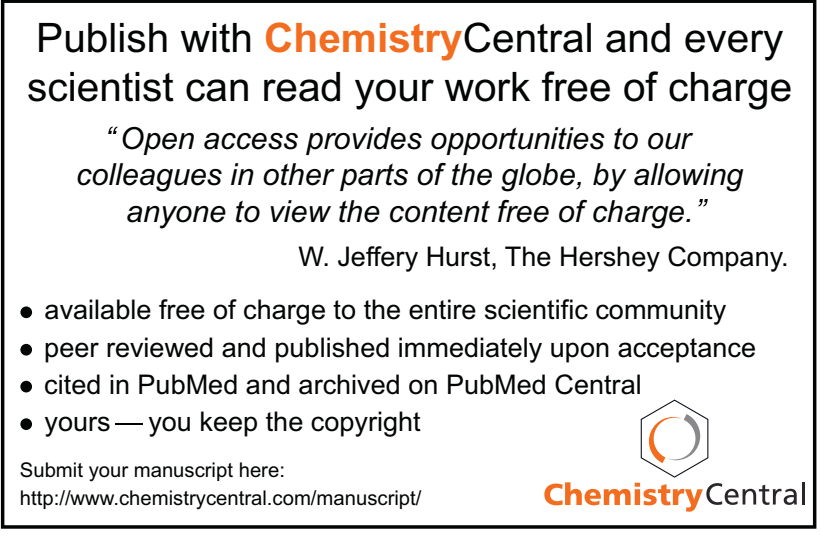

